\title{
Las habilidades cognitivas en el profesional de la Información desde la perspectiva de proyectos y asociaciones internacionales
}

\author{
Maylín Frías Guzmán* \\ Yinet Haro Águila** \\ Iliana Artiles Olivera***
}

Artículo recibido:

21 de mayo de 2014.

Artículo aceptado: 5 de noviembre de 2016.

\section{Resumen}

La sociedad de la información impone retos a los profesionales del mismo campo que influyen en la concepción de los programas y documentos rectores de su formación. El presente artículo expone aspectos teóricos conceptuales relativos a las habilidades cognitivas. Se analizan directrices internacionales de la Special Library Association (SLA), el proyecto DECIDoc, la Agencia Nacional de Evaluación de la Calidad y Acreditación (ANECA) y el European Council of Information Associations (ECIA). La investigación documental

* Profesora Auxiliar. Departamento de Ciencias de la información, Facultad de Ingeniería Industrial y Turismo, Universidad Central "Marta Abreu" de Las Villas (UCLV). maylinfg@uclv.edu.cu

** Profesora. Preuniversitario Vocacional de Ciencias Exactas "Ernesto Ché Guevara". yinetah@ce.vc.rimed.cu

$\star \star \star$ Profesora Titular. Vicerrectorado Docente. Universidad Central "Marta Abreu" de Las Villas (UCLV). liao@uclv.edu.cu

INVESTIGACIÓN BIBLIOTECOLÓGICA, vol. 31, núm. 71, enero/abril, 2017, México, ISSN: 0187-358X pp. 201-218 
que se presenta realiza un análisis de contenido desde un enfoque cualitativo que permitió identificar las habilidades cognitivas de análisis, síntesis, aplicación y evaluación como las más representativas en la formación del profesional de la información.

Palabras clave: Habilidades cognitivas; Ciencias de la información; Formación del profesional de la información

\section{Abstract}

Librarians' Cognitive Skills from the Perspective of International Projects and Library Associations Maylín Frías Guzmán, Yinet Haro Águila, and Iliana Artiles Olivera

The Information Society imposes challenges to information professionals who influence the design of syllabi and associated curricular documents. This article presents some conceptual-theoretical reflections on cognitive skills. International guidelines of the Special Library Association (SLA), the DECIDoc project, the National Agency for Quality and Accreditation Assessment (ANECA) and the European Council of Information Associations (ECIA) are also analyzed. The documentary research presented entails a qualitative content analysis showing that the most representative cognitive skills in the formation of information professionals are analysis, synthesis, application, and evaluation.

Keywords: Cognitive Skills; Information Sciences; Information Professional Training

\section{Introducción}

$\mathrm{L}$ os vertiginosos cambios económicos, políticos, sociales, intelectuales, científicos y tecnológicos que se evidencian en la actualidad exigen individuos competentes y preparados para asumir el reto que trae consigo estar inmersos en la sociedad de la información y el conocimiento. Diversos teóricos la definen como una gran revolución científica y de avances tecnológicos que presuponen cambios en las personas. Las transformaciones exigen: 
Individuos reflexivos que comprendan la información, la evalúen y actúen sobre ella; pero también individuos que generen muchas ideas variadas y originales [que] creen un entorno social caracterizado por el bienestar, la justicia y la equidad: una meta atractiva y ambiciosa que tienen que llevar a cabo los estudiantes en formación. ${ }^{1}$

En el ámbito de la Bibliotecología y la Ciencia de la Información, el trabajo no finaliza poniendo en práctica, solamente, lo que Ponjuan definiera como "las fortalezas de la profesión". ${ }^{2}$ No basta con revolucionar las formas de recolectar, procesar, almacenar y diseminar la información; sino cuando se cuestiona la información y la realidad social.

La información como objeto de trabajo de la profesión establece relaciones interdisciplinarias y multidisciplinarias, que derivan en la inserción de los graduados en múltiples esferas donde el uso adecuado y eficiente de datos contextualizados es clave para la supervivencia profesional. En este sentido, las habilidades cognitivas ameritan una atención especial como base para el desarrollo de las demás.

En la literatura referida a la formación del profesional de la información, las habilidades o competencias cognitivas son identificadas como esenciales en el perfil profesional. Los estudios de Castro y Chávez, 2012; Escalona et al., 2008; Pirela y Peña 2005, Oliva, 2012; Pirela, 2007; Pirela y Cortés, 2014, aluden indistintamente a la incorporación de las mismas en los currículos de Bibliotecología y Ciencia de la Información.

El presente trabajo pretende identificar las habilidades cognitivas en los documentos que intentan establecer un consenso de competencias o habilidades en la formación del profesional de la información en el ámbito de la educación superior. El estudio ofrecerá bases para la inserción de las habilidades cognitivas en el proceso formativo del profesional de la Información a partir de la familiarización con documentos normativos. Contribuirá a encauzar la formación de profesionales competentes y capaces de enfrentar los cambios económicos, políticos, sociales y culturales que ocurren vertiginosamente.

1 M. Sanz, Uso pedagógico de recursos y herramientas de Internet (Madrid III Congreso Internacional EducaRed: 2005), 9.

2 G. Ponjuan, Gestión de información en las organizaciones: principios, conceptos y aplicaciones (Santiago de Chile, 1998), 12. 


\section{Metodología}

El estudio que se presenta corresponde a una investigación documental desde un enfoque cualitativo. Se caracterizó por el uso de fuentes de información documentales para su realización. La elección de las fuentes se realizó con base en la correspondencia entre el tema investigado y el contenido de los documentos recopilados. Se seleccionaron documentos reconocidos a nivel internacional en la formulación de directrices para la formación de los profesionales de la información.

Se empleó el método de análisis de contenido: las etapas en las que se procedió en su aplicación correspondieron con el criterio de Gaitán y Piñuel. ${ }^{3}$ Se definieron los elementos sobre los que recae el análisis según Hernández et al. ${ }^{4}$

1) Selección de la comunicación. Se estableció como universo los siguientes documentos:

- "Competencias para profesionales de la información del siglo XXI": documento elaborado por la Special Library Association (SLA), asociación internacional que acoge bibliotecarios y documentalistas del entorno corporativo académico y gubernamental.

- "Euroguide LIS: The Guide to Competencies for European Professionals in Library and Information Services": documento elaborado como parte del proyecto DECIDoc, subvencionado por el Programa Leonardo da Vinci de la Unión Europea, en el que participaron nueve asociaciones profesionales europeas.

- Libro blanco: título de grado en Información y Documentación: proyecto desarrollado por la Agencia Nacional de Evaluación de la Calidad y Acreditación (ANECA).

- “Eurorreferencial en Información y Documentación": resultado del perfeccionamiento del Euroguide LIS, creado por el European Council of Information Associations (ECIA)

2) Selección de las categorías de análisis:

- Categorías: habilidades cognitivas entendidas como:

las habilidades cognitivas son aquellas que permiten al individuo conocer, pensar, almacenar información, organizarla y transformarla hasta generar nuevos productos, realizar operaciones tales como establecer

3 J. A. Gaitán Moya y J. L. Piñuel Raigda, Técnicas de Investigación en Comunicación Social: elaboración y registro de datos (Madrid: Síntesis, 1998).

4 R. Sampieri Hernández et al., Metodología de la Investigación (México: McGraw Hill, 2006). 
relaciones, formular generalizaciones, tomar determinaciones, resolver problemas y lograr aprendizajes perdurables y significativos. ${ }^{5}$

- Subcategorías de análisis: recordar, entender, aplicar, analizar, sintetizar y evaluar a partir de la taxonomía de Bloom revisada.

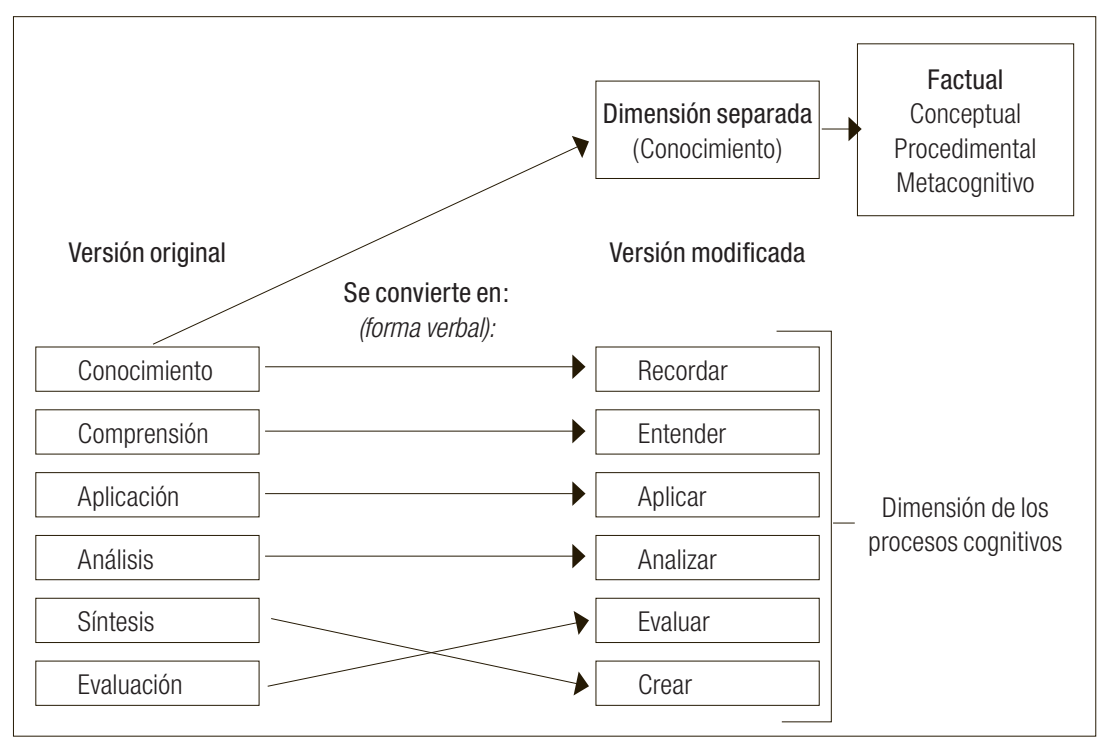

Figura 1. Modificaciones estructurales a la Taxonomía de Bloom Revisada.

Fuente: Vásquez, 2010.

3) Selección de las unidades de análisis: Las palabras constituyeron las unidades de análisis seleccionadas en el presente estudio. Los términos empleados para expresar las habilidades cognitivas fueron identificados en los enunciados que describen las competencias en cada uno de los documentos.

\section{Las habilidades cognitivas}

Las primeras referencias teóricas sobre las habilidades cognitivas (cognitive skills) y habilidades para pensar (thinking skills) aparecieron en el mundo anglosajón durante la década de los cincuenta.

5 S. Schmidt, Competencias, habilidades cognitivas, destrezas prácticas y actitudes definiciones y desarrollo (2006): 2, https://rmauricioaceves.files.wordpress.com/2013/02/definicion -comphabdestrezas.pdf. 
Su génesis se ubica en el campo de la psicología cognitiva, entendiéndose como las operaciones del pensamiento por medio de las cuales el sujeto se apropia de los contenidos y del proceso que usó para ello.

Para Vásquez el desarrollo histórico de los estudios sobre habilidades cognitivas se produce:

A partir de la década de 1990 surge un renovado interés en el estudio de los procesos cognitivos, pero esta vez desde una óptica distinta a la de los años ' 60 y '70. Dentro de esta nueva corriente teórica, destaca el interés en las habilidades para pensar (thinking skills), las que pueden ser definidas como ciertas capacidades mentales que permiten a las personas captar, procesar e interpretar información, y que pueden ser enseñadas. ${ }^{6}$

En el ámbito psicológico se considera que la expresión de las habilidades del pensamiento requiere de las estructuras cognitivas utilizadas por las personas para realizar las operaciones mentales. El desarrollo de tales estructuras no constituye un proceso espontáneo, sino que debe ser estimulado y ejercitado a través de experiencias y/o de entrenamiento formal o informal. Estas habilidades se asocian al proceso del pensamiento y constituyen las formas básicas para el aprendizaje. Se definen como operaciones, procedimientos, procesos, facultades, destrezas que transforman la información en conocimiento.

En el marco del diseño curricular, los pedagogos españoles Coll, Del Carmen, Zabala, Mauri, entre otros, distinguen las capacidades cognitivas como esenciales en la formación de los estudiantes. ${ }^{7}$ Sanz $^{8}$ destaca la necesidad de convertirlas en el objetivo prioritario del sistema educativo actual.

La creación de los modelos conceptuales sobre habilidades cognitivas tuvo sus inicios en la década de los cincuenta del siglo XX. Su aparición estuvo condicionada por la necesidad de un sistema de clasificación teórico que posibilitara la medición del coeficiente intelectual, hasta entonces examinado por test con dimensiones insuficientes. Los modelos más reconocidos fueron:

- La estructura del intelecto de J.P. Guilford (1967).

- Taxonomía de objetivos educativos de Bloom (1956).

6 A. Vázquez Córdoba, "Competencias Cognitivas en la Educación Superior", Revista Electrónica de Desarrollo de Competencias (REDEC) 2, no. 6 (2010): 40.

7 E. Ander-Egg, La planificación educativa: conceptos, métodos, estrategias y técnicas para educadores (La Plata: Magisterio del Río de La Plata, 1993).

8 M. L. Sanz de Acedo Lizarraga, Competencias Cognitivas en Educación Superior (Madrid, 2010). 
- Taxonomía de Bloom revisada de Krathwohl (2002).

- Taxonomía de habilidades del pensamiento crítico de Halpern (1994).

En el modelo de Guilford, la habilidad cognitiva es el resultado de la combinación de las tres dimensiones esenciales a partir de las cuales se describe el pensamiento: operaciones, contenido y producto. El cruce de las categorías de cada dimensión da lugar a varias combinaciones que hacen flexible el modelo. ${ }^{9}$

El modelo de Bloom organiza de forma jerárquica un conjunto de habilidades intelectuales según su complejidad creciente. Según Vásquez, ${ }^{10}$ la taxonomía fue aplicada en el ámbito educativo para clasificar objetivos y metas educativas. Señala los tipos de tareas que debe resolver un individuo (o un estudiante) para su logro y entregando definiciones operacionales para poner en práctica habilidades en cada una de seis categorías del ámbito cognitivo (conocimiento, comprensión, aplicación, análisis, síntesis y evaluación).

Varios pedagogos consideran que los currículos, programas o instrumentos de buena calidad serán aquellos que estimulen el trabajo de los estudiantes a través de los distintos niveles cognitivos. Para Herrera, et al. ${ }^{11}$ es necesaria la inclusión de las expresiones de la Taxonomía de Bloom en su declaración. Sobre el asunto expresan: "para que se dé un aprendizaje debe existir conocimiento, comprensión, análisis, síntesis, aplicación y valoración”.

La taxonomía de Bloom revisada asume un carácter bidimensional, pues sus revisores decidieron distinguir entre conocimiento como el proceso de recordar y conocimiento como contenido que es recordado. En la nueva propuesta se incluye una clasificación del conocimiento como contenido del aprendizaje. Se establece una distinción entre los diferentes tipos de conocimiento e introduce el concepto de metacognición.

El modelo de Halpern estableció un esquema conceptual de habilidades de pensamiento crítico. Halpern ${ }^{12}$ planea que el pensamiento crítico se basa en el uso de las habilidades cognitivas con el objetivo de incrementar las probabilidades de obtener resultado deseable pues este pensamiento tiene un propósito marcado, es razonado, dirigido a metas y está orientado a resolver problemas, formar inferencias y tomar decisiones. Cuando se piensa críticamente se evalúan los resultados de los procesos del pensamiento.

La aplicación de los modelos al ámbito digital dio origen a la llamada Ta-

9 J. P. Guilford, "Creativity Research: Past, Present and Future", en Frontiers of Creativity Research: Beyond The Basics, ed. S. G. Isaksen (New York, 1987), 33-65.

10 A. Vázquez Córdoba, "Competencias Cognitivas en la Educación Superior", 34-64.

11 F. Herrera Clavero et al., "Actualidad del diseño curricular", Euphoros, no. 5 (2002): 147.

12 D. F. Halpern, Thought and Knowledge: An Introduction to Critical Thinking (New Jersey: Lawrence Erlbaum Associates, 2003). 
xonomía de Bloom para la era digital de Andrew Churches. El autor intenta abarcar los procesos cognitivos que se producen asociados a las Tecnologías de la Información y la Comunicación (TIC).

Según Vásquez, la Taxonomía de Bloom de Anderson y Krathwohl, y el modelo teórico de Halpern se encuentran entre los marcos teórico-conceptuales más valiosos en la actualidad:

El primero como una sólida herramienta para clasificar objetivos educacionales en el diseño curricular y didáctico, en diversos niveles educativos incluyendo el superior o universitario, y el segundo como un detallado marco de análisis del pensamiento y habilidades cognitivas de orden superior, entre ellas la de pensamiento crítico. ${ }^{13}$

Con base en estos modelos se ha derivado una diversidad de clasificaciones de habilidades cognitivas y términos para expresar las acciones que comprenden.

La presente investigación asume como modelo de habilidades cognitivas el establecido en la Taxonomía de Bloom revisada. El ámbito de aplicación y su alcance se ajustan a los fines que se persiguen.

\section{La formación de habilidades cognitivas desde la perspectiva de proyectos $y$ asociaciones internacionales en el ámbito de la Bibliotecología y \\ Ciencia de la Información}

Las directrices sobre programas educativos y textos sobre competencias o habilidades profesionales, desarrollados por diversas instituciones o asociaciones profesionales de la información en el mundo, aluden a la formación de habilidades cognitivas. Su inclusión se distribuye, indistintamente, en la tipología de genéricas o específicas.

En el listado de las 23 competencias que establece el Euroguide LIS "las diez primeras son propiamente de formación teórica y práctica en la disciplina; las restantes o son competencias de tipo instrumental o pertenecen a otras disciplinas, como el marketing, la gestión administrativa o las técnicas de edición". ${ }^{14}$ Dentro de las competencias profesionales que enuncia el documento la formación de habilidades cognitivas es esencial en los aspectos vinculados al:

13 A. Vázquez Córdoba, "Competencias Cognitivas en la Educación Superior", 63.

14 ASLIB, "Euroguide LIS: The Guide to Competencies for European Professionals in Library and Information Services”, Madrid, 2000, 8, última actualización 6 de enero, 2013, http:// www.aslib.co.uk/pubs/2001/18/01/foreword.htm. 
- conocimiento del entorno profesional de la información y la documentación;

- conocimiento del marco jurídico y administrativo nacional e internacional de la gestión de la información;

- análisis y representación de la información. ${ }^{15}$

Las dos primeras requieren de la ejercitación de los procesos del pensamiento para la adquisición del conocimiento que se menciona. La tercera está vinculada con la esfera de organización y representación de la información donde la distinción, diagramación, delimitación de los elementos informativo-documentales son básicos para un desempeño profesional adecuado.

En las competencias transversales que se desglosan, se alude a la capacidad de análisis y síntesis como base para el desempeño profesional. El conocimiento de una lengua extranjera implica recordar y entender. Los conocimientos de informática relativos al ámbito de estudio se vinculan al dominio de la concepción de herramientas tecnológicas asociadas al ámbito informacional. El razonamiento crítico apunta a la formación de los procedimientos deevaluación.

En las competencias para los profesionales de la información en el siglo XXI, que declara la Asociación de Bibliotecas Especializadas (SLA) se parte del presupuesto que "las oportunidades emergentes continuas impulsaran a los profesionales preparados a escenarios aún no vistos de recuperación avanzada de información con nuevos escenarios de cooperación, interpretación, síntesis, desarrollo de productos y servicios virtuales a escala global". ${ }^{16}$ Las habilidades cognitivas no se excluyen de la propuesta de competencias profesionales, personales y básicas que declara el documento.

Estas últimas se expresan en la contribución al conocimiento de la profesión y el aprendizaje, donde las habilidades cognitivas, en su totalidad, son vitales. Las personales se identifican esencialmente en el momento en que el profesional, en un contexto específico, "piensa con innovación y creativamente; busca oportunidades nuevas o las reinventa" ${ }^{17}$

Las competencias profesionales "están relacionadas con el conocimiento de los recursos, acceso, tecnología y administración, y la habilidad para utilizar este conocimiento como la base para proveer servicios informativos

15 ASLIB, "Euroguide LIS: The Guide to Competencies for European Professionals in Library and Information Services", 8.

16 Abels et al., Competencias para profesionales de la información del siglo XXI, 9.

17 Ibid., 9. 
de la más alta calidad" ${ }^{18}$ Se subdividen en cuatro específicas que enuncian habilidades:

a) Administración de organizaciones informativas

b) Administración de recursos informativos

c) Administración de servicios informativos

d) Aplicación de herramientas y tecnologías informativas

En las dos primeras la evaluación se enuncia expresamente. La "a" refiere que el desempeño organizacional necesita de la apreciación, diagnóstico, y peritaje de la organización, los servicios, los productos y las políticas informativas. La "b" asocia la habilidad para evaluar críticamente los recursos de información.

En la administración de los servicios, aplicar adquiere connotación en la supervisión de los servicios a través del empleo de lineamientos apropiados y el liderazgo de procesos de aprendizaje y autoaprendizaje. Analizar y sintetizar se vinculan a la transformación de información para dar "respuestas confiables o información operable para clientes". ${ }^{19}$

La aplicación de herramientas y tecnologías informativas desde su enunciado explicita la habilidad cognitiva de aplicar. Se relaciona a los aspectos de organización, representación y recuperación de la información.

La Agencia Nacional de Evaluación de la Calidad y Acreditación (ANECA) de España, integra un conjunto de competencias, resultado de propuestas anteriores, en el Libro blanco: título de grado en Información y Documentación. En el mismo, las habilidades cognitivas se manifiestan tanto en las competencias transversales como específicas que se declaran.

Las competencias específicas se dividen en conocimientos, competencias profesionales y académicas. De manera implícita la adquisición de los conocimientos que se declaran para la titulación conllevan a la formación de la habilidad de conocer. En el resto, las habilidades cognitivas se manifiestan esencialmente como se muestra en la Tabla 1. 
Tabla 1. Competencias profesionales y académicas vinculadas a las habilidades cognitivas en el Libro blanco.

\begin{tabular}{|c|c|c|}
\hline \multirow{8}{*}{$\begin{array}{l}\text { Competencias } \\
\text { específicas }\end{array}$} & \multirow{3}{*}{$\begin{array}{l}\text { Competencias } \\
\text { profesionales }\end{array}$} & $\begin{array}{l}\text { Habilidades en el manejo de las tecnologías como medio } \\
\text { indispensable en los procesos de tratamiento y transferencia } \\
\text { de la información. }\end{array}$ \\
\hline & & $\begin{array}{l}\text { Habilidades para analizar, asesorar y formar a productores, } \\
\text { usuarios y clientes de servicios de información, así como, } \\
\text { habilidades en los procesos de negociación y comunicación. }\end{array}$ \\
\hline & & $\begin{array}{l}\text { Habilidades en la obtención, tratamiento e interpretación de datos } \\
\text { sobre el entorno de las unidades y servicios de información; el } \\
\text { estudio, la gestión, la evaluación de los procesos de producción, } \\
\text { transferencia, uso de la información y de la actividad científica. }\end{array}$ \\
\hline & \multirow{5}{*}{$\begin{array}{l}\text { Competencias } \\
\text { académicas }\end{array}$} & $\begin{array}{l}\text { Comprender y aplicar los principios y las técnicas para la } \\
\text { planificación, organización y evaluación de sistemas, unidades } \\
\text { y de servicios de información. }\end{array}$ \\
\hline & & $\begin{array}{l}\text { Comprender y aplicar los principios y las técnicas para la } \\
\text { reunión, selección, organización, representación, preservación, } \\
\text { recuperación, acceso, difusión e intercambio de la información. }\end{array}$ \\
\hline & & $\begin{array}{l}\text { Utilizar y aplicar herramientas informáticas para la implantación, } \\
\text { desarrollo y explotación de sistemas de información. }\end{array}$ \\
\hline & & $\begin{array}{l}\text { Comprender y aplicar las técnicas de evaluación de las fuentes } \\
\text { y recursos de información. }\end{array}$ \\
\hline & & $\begin{array}{l}\text { Comprender y aplicar las técnicas de gestión y marketing de } \\
\text { sistemas, unidades y servicios de información }\end{array}$ \\
\hline & & \\
\hline
\end{tabular}

En la muestra se exponen las habilidades de aplicar, analizar y comprender. La primera se reitera y vincula con las técnicas de planificación, organización y evaluación de sistemas, unidades y servicios de información, así como, con las técnicas, las normativas y otros instrumentos utilizados en la selección, organización, representación, preservación, recuperación, acceso, difusión e intercambio de la información. Las habilidades de conocer y crear subyacen a partir de la obtención y la misma aplicación de la información o herramientas tecnológicas en los diferentes escenarios informacionales.

En las competencias transversales se dividen en instrumentales, personales y sistemáticas. En las primeras, las habilidades cognitivas se enuncian explícitamente asociadas a la "capacidad de análisis y de síntesis aplicadas a la 
gestión y organización de la información”. Las personales las vinculan a partir del "razonamiento crítico en el análisis y la valoración de alternativas. ${ }^{20}$

En las últimas, la "capacidad para el aprendizaje autónomo" implica el desarrollo de habilidades del pensamiento. Sin embargo, se alude a habilidades de toma de decisiones y resolución de problemas y de pensamiento creativo según el modelo de Halpern, 2003 cuando se enuncian:

- Capacidad para emprender mejoras y proponer innovaciones.

- Capacidad de dirección y liderazgo. ${ }^{21}$

El Eurorreferencial en Información y Documentación declara 33 campos de competencias divididos en los siguientes grupos:

- Grupo I - Información.

- Grupo T-Tecnologías.

- Grupo C-Comunicación.

- Grupo G-Gestión.

- Grupo S - Otros saberes. ${ }^{22}$

El documento considera competente al profesional que demuestre lo establecido cada uno de los cuatro niveles que declara. Esta aclaración es válida porque se puede establecer una correspondencia con los niveles de funcionamiento del sistema cognitivo humano que establece Sanz ${ }^{23}$ (Véase Tabla 4).

Tabla 2. Correspondencia entre los niveles de funcionamiento del sistema cognitivo humano (Sanz, 2010) y los niveles de competencias del Eurorreferencial.

\begin{tabular}{|l|l|l|}
\hline \multicolumn{2}{|c|}{$\begin{array}{c}\text { Niveles de funcionamiento del } \\
\text { sistema cognitivo humano }\end{array}$} & Niveles de competencias del eurorreferencial \\
\hline \multirow{2}{*}{ Nivel bajo } & $\begin{array}{l}\text { Sensación } \\
\text { Percepción } \\
\text { Atención }\end{array}$ & \\
\hline
\end{tabular}

20 ANECA, Libro blanco: título de grado en Información y Documentación (Madrid: Omán Impresores, 2004), 50.

21 Ibid., 50.

22 ECIA, Eurorreferencial en Información y Documentación. 2004, 9-10.

23 Sanz de Acedo Lizarraga, Competencias Cognitivas en Educación Superior. 


\begin{tabular}{|c|c|c|c|}
\hline \multirow{2}{*}{ Nivel medio } & \multirow{2}{*}{$\begin{array}{l}\text { Aprendizaje } \\
\text { Memoria } \\
\text { Comunicación }\end{array}$} & Nivel 1 & Sensibilización \\
\hline & & Nivel 2 & Conocimiento de las prácticas. \\
\hline \multirow{2}{*}{ Nivel alto } & \multirow{2}{*}{$\begin{array}{l}\text { Razonamiento } \\
\text { Creatividad } \\
\text { Toma de decisiones } \\
\text { Resolver problemas }\end{array}$} & Nivel 3 & Dominio de las herramientas \\
\hline & & Nivel 4 & Dominio metodológico \\
\hline
\end{tabular}

Las habilidades cognitivas de recordar y entender están básicamente presentes en los niveles de sensibilización y de conocimiento de las prácticas. En éstas, los profesionales se conforman con usar las herramientas y cuenta con un instrumento de lectura y representación de los fenómenos estudiados, respectivamente.

Aplicar, analizar y evaluar se vinculan más al dominio de las herramientas y las metodologías teniendo en cuenta que

El profesional conoce las técnicas y su contenido, sabe definirlas, hablar de ellas y también controla su empleo. Puede interpretar una situación y emitir un juicio que implique que una tarea sea adaptada ó idear una herramienta. Puede escoger actos elementales y encadenarlos a acciones complejas. El profesional emplea una determinada técnica, pero puede aplicarla en otras situaciones, transferirla a otros procesos, encontrarle otros campos de aplicación, crear mejoras e incluso otras técnicas más elaboradas o adaptadas. Puede crear nuevas herramientas o nuevos productos, tener un acercamiento estratégico o global hacia su actividad. El profesional se da cuenta de la complejidad de las situaciones e intenta buscar soluciones originales adecuadas. ${ }^{24}$

En el Grupo I- Información las habilidades cognitivas se expresan en:

- comprensión y atención a las necesidades de información;

- aplicación de las disposiciones y los procedimientos legales y reglamentarios relativos a la actividad de información y documentación;

- elaboración de modelos, crear y explotar metadatos y accesos;

- evaluación, selección y validación de fuentes de información;

- evaluación de la adecuación entre la demanda y la respuesta proporcionada. 
- Elaboración y aplicación de criterios de selección, de adquisición, de conservación y de eliminación de documentos.

- Definición y aplicación de políticas de desarrollo de los fondos y colecciones.

- Aplicación de métodos, medidas y técnicas destinadas a ordenar, proteger, conservar, restaurar y comunicar al público soportes documentales.

En el Grupo T-Tecnologías el conocimiento y aplicación de las tecnologías de la información en los contextos informacionales es fundamental, sin obviar los procesos de análisis y evaluación de la disposición de la información en el ámbito digital y el uso de las herramientas adecuadas para la resolución de problemas específicos.

En el Grupo C-Comunicación, son inherentes las habilidades cognitivas a partir de los procesos de percepción, memorización, comprensión y creación de información para establecer un intercambio con otros sujetos.

En esta área se establece una relación con la figura del usuario, como sujeto que conoce, genera y usa información. Desde una perspectiva cognitiva se explica quién es y cómo se vincula con la información. Según Hernández, ${ }^{25}$ "considerar los procesos mentales para explicar fenómenos referidos a la información conlleva directamente a aplicar sus principios al área de usuarios", así como a los "campos de investigación como la clasificación o el diseño de sistemas de recuperación de información".

En el Grupo G-Gestión, se evidencian en la comprensión de los aspectos teóricos relativos a la gestión de información y el conocimiento. En los escenarios informacionales la aplicación de herramientas gerenciales para auxiliar la toma de decisiones y resolución de problemas en el ámbito empresarial.

En el Grupo S-otros saberes se integran las habilidades cognitivas en la adquisición de conocimientos complementarios de otras ramas del conocimiento. Podemos destacar que dentro de las 20 aptitudes que declara el Eurorreferencial se declara el Análisis como una de ellas. En este sentido se alude a lo siguiente.

1) (Espíritu de) análisis: reconocer los elementos específicos o característicos de una situación o de un problema, dentro de un documento (sin importar su formato), de una relación de datos o de una petición.

25 P. Hernández Salazar, "El fenómeno usuarios de la información desde un enfoque cognitivo", Investigación Bibliotecológica 27, no. 61 (2013): 122. 
Dividir dichos elementos en diferentes categorías. Establecer entre ellos relaciones de causalidad o de interdependencia y determinar su significado.

2) (Espíritu) Crítico: emitir un juicio de valor sobre una declaración, un documento, una persona, una organización, un método de trabajo, una técnica documental, etc., detectando con objetividad los puntos fuertes y los débiles. Ser capaz de comparar una información, por ejemplo en cuanto a su veracidad o a la fiabilidad de sus fuentes. ${ }^{26}$

De manera general, los documentos relativos a la formación de profesionales de la información incluyen las habilidades cognitivas implícita y explícitamente según se resume a continuación:

- El proyecto DECIDoc incluye las habilidades cognitivas de análisis, síntesis y entendimiento para la adquisición del conocimiento relativo al entorno profesional, el marco jurídico y administrativo nacional e internacional de la gestión, así como, la organización y representación de la información. La evaluación se manifiesta en la exigencia de un razonamiento crítico ante los problemas profesionales.

- La Asociación de Bibliotecas Especializadas (SLA) enfatiza en la evaluación en el ámbito de la administración las organizaciones informativas y los recursos informativos. Aplicar adquiere connotación en la administración de los servicios informativos y el desarrollo de herramientas y tecnologías informativas.

- La ANECA de España, integra las habilidades cognitivas de crear, aplicar, analizar y comprender en las técnicas de planificación, organización y evaluación de sistemas, unidades y servicios de información, así como, en las técnicas, normativas y otros instrumentos utilizados en la selección, organización, representación, preservación, recuperación, acceso, difusión e intercambio de la información. Alude a habilidades de toma de decisiones y resolución de problemas y de pensamiento creativo a través del desarrollo de capacidades para emprender mejoras, proponer innovaciones, dirigir y liderar

- El Eurorreferencial establece las habilidades cognitivas de recordar y entender, básicamente, en los niveles de sensibilización y de conocimiento de las prácticas. Aplicar, analizar y evaluar se vinculan más al dominio de las herramientas y las metodologías utilizadas en la profesión por lo se expresan en los grupos de Tecnologías, Comunicación y 
Gestión. El espíritu de análisis y el espíritu crítico se contemplan en las aptitudes para la toma de decisiones y resolución de problemas, así como, para la evaluación de recursos de información.

\section{Conclusiones}

- Los documentos relativos a la formación de profesionales de la información incluyen las habilidades cognitivas en el marco de las competencias que declaran, enfatizando en analizar, sintetizar, aplicar y evaluar en detrimento de recordar. Favorecen el desarrollo del pensamiento comprensivo, crítico y analítico en función de los campos de actuación del profesional relativos a la selección, organización, representación y difusión de información.

- Se muestran habilidades cognitivas tanto de manera explícita: diseñar, identificar y evaluar, así como, implícita: comprender, analizar y sintetizar vinculadas al tratamiento de la información. La aplicación y evaluación se subrayan en los procesos de gestión de la información, generación, transferencia del conocimiento y aprendizaje organizacional y social. Sintetizar se encuentra presente en la medida que el profesional sea capaz de traducir del lenguaje natural al lenguaje documental y utilizar la información de modo tal que le ofrezca a las organizaciones y usuarios un mejor acceso y uso.

\section{Referencias}

Abelsm E., R. Jones, J. Latham, D. Magnoni y J. Gard Marshall. Competencias para profesionales de la información del siglo XXI, 2003.

Ander-Egg, E. La planificación educativa: conceptos, métodos, estrategias y técnicas para educadores. La Plata: Editorial Magisterio del Río de La Plata, 1993.

ANECA. Libro blanco: título de grado en Información y Documentación. Madrid: Omán Impresores, 2004.

ASLIB. "Euroguide LIS: The Guide to Competencies for European Professionals in Library and Information Services". Madrid, 2000. Última actualización 6 de enero, 2013. http://www.aslib. co.uk/pubs/2001/18/01/foreword.htm.

Castro Viguera, Y. y Y. Chávez Montejo. "La interdisciplinariedad en la formación profesional del analista de información: propuesta de competencias Ciencias de la Información”. Ciencias 43, no. 2 (2012): 55-59. 
ECIA. "Eurorreferencial en Información y Documentación: competencias y aptitudes de los profesionales europeos de información y documentación 1”. Madrid: SEDIC, 2004.

Escalona Ríos, L., R. Arriola Ruiz y R. A. Bautista. "El enfoque de competencias en la formación de recursos humanos para bibliotecas". Investigación Bibliotecológica 22, no. 46 (2008): 15-40. http://www. scielo.org.mx/scielo.php?script=sci_arttext\&pid=S0187358X2008000300002\&lng=es\&nrm=iso.

Gaitán Moya, J. A. y J. L. Piñuel Raigda. Técnicas de Investigación en Comunicación Social: elaboración y registro de datos. Madrid: Síntesis, 1998.

Guilford, J. P. "Creativity Research: Past, Present and Future”. En Frontiers of Creativity Research: Beyond the Basics, editado por S. G. Isaksen, 33-65. Nueva York, 1987.

Halpern, D. F. Thought and Knowledge: An Introduction to Critical Thinking. New Jersey: Lawrence Erlbaum Associates, 2003.

Hernández Salazar, P. "El fenómeno usuarios de la información desde un enfoque cognitivo". Investigación Bibliotecológica 27, no. 61 (2013):107-131.

Hernández Sampieri, R., C. Fernández-Collado y Pilar Baptista Lucio. Metodología de la Investigación. México: McGraw Hill, 2006.

Herrera Clavero, F., M. I. Herrera Ramírez y M. I. Ramírez Salguero. "Actualidad del diseño curricular". Euphoros, no. 5 (2002): 141172.

Krathwohl, D. A. "Revision of Bloom's Taxonomy". Theory into Practice 41, no. 4 (2002): 212-218.

Oliva Marañón, C. "Competencias y formación universitaria del documentalista en el Espacio Europeo de Educación Superior (EEES): estudio de su adecuación al entorno laboral". Investigación Bibliotecológica 26, no. 58 (2012): 15-45. http://www.scielo.org.mx/scielo. php? script=sci_arttext $\&$ pid $=$ S0187-358X2012000300002\&lng= es\&nrm=iso.

Pirela Morillo, J. "Las tendencias educativas del siglo XXI y el currículo de las escuelas de Bibliotecología, Archivología y Ciencia de la Información de México y Venezuela". Investigación Bibliotecológica 21, no. 43 (2007): 73-105. http://www.scielo.org.mx/scielo. php? script $=$ sci_arttext $\&$ pid $=$ S0187-358X2007000200004\&ln$\mathrm{g}=\mathrm{es} \& \mathrm{nrm}=$ iso.

Pirela Morillo, J. y J. D. Cortés Vera. "El desarrollo de competencias informacionales en estudiantes universitarios. Experiencia y perspectivas en dos universidades latinoamericanas". Investigación Bibliotecológica 28, no. 64, (2014): 145-172. http://www.scielo.org.mx/ scielo.php?script=sci_arttext\&pid=S0187358X2014000300008\&lng=es\&nrm=iso.

Pirela Morillo, J. y T. Peña Vera. "Nuevos desafíos para la formación del profesional de la información frente al surgimiento de la cibersociedad: un enfoque de competencias". Investigación Bibliotecológica 
19, no. 38 (2005): 118-139. http://www.scielo.org.mx/scielo.php? script $=$ sci_arttext \&pid $=$ S0187-358X2005000100007\&lng =es \& nrm=iso.

Ponjuan, G. Gestión de información en las organizaciones: principios, conceptos y aplicaciones. Santiago de Chile, 1998.

Sanz de Acedo Lizarraga, M. L. Competencias Cognitivas en Educación Superior. Madrid, 2010.

Sanz, M. Uso pedagógico de recursos y herramientas de Internet. III Congreso Internacional EducaRed. Madrid, 2005.

Schmidt, S. Competencias, habilidades cognitivas, destrezas prácticas y actitudes definiciones $y$ desarrollo. https://rmauricioaceves.files. wordpress.com/2013/02/definicion-comphabdestrezas.pdf.

Vásquez Cordoba, A. "Competencias Cognitivas en la Educación Superior". Revista Electrónica de Desarrollo de Competencias (REDEC) 2, no. 6 (2010): 34- 64.

Para citar este artículo:

Frías Guzmán, Maylín, Yinet Haro Águila e Iliana Artíles Olivera. "Las habilidades cognitivas en el profesional de la Información desde la perspectiva de proyectos y asociaciones internacionales". Investigación Bibliotecológica 31, no. 71 (enero-abril 2017): 201-218. DOI.htpp://dx.doi.org/10.22201/iibi.0187358xp.2017.71.57816 